\title{
Erratum to: Social media-based systems: an emerging area of information systems research and practice
}

\author{
Gohar Feroz Khan
}

Published online: 30 November 2012

(C) Akadémiai Kiadó, Budapest, Hungary 2012

\section{Erratum to: Scientometrics \\ DOI 10.1007/s11192-012-0831-5}

Error in the footer of Table 5 in the original publication of the article is corrected below. The complete paragraph that has appeared as a footnote to Table 5, but should read beneath the header "Technical aspect of SMS" is provided for your reading.

\section{Technical aspect of SMS}

Technical stream of the SMS research looks into the technical aspect of SMS in organizational and non-organizational context (see Fig. 2 stream 5 and Tables 5, 6). Researchers discuss issues, such as, design, architecture, and algorithms (Alon et al. 2010; Rodriguez et al. 2006; Caverlee et al. 2010; Allen et al. 2010; Shekarpour and Katebi 2010; Shapira and Zabar 2011) related to SMS, for example, clustering algorithms (Bar-Yossef et al. 2006), algorithms for extracting inter-firm networks over web (Jin et al. 2008). Other topics discussed are user modeling and sentiments (Liu et al. 2006), reliability (Korfiatis et al. 2006), and privacy and security (Chi et al. 2010) issues related to the SMS, for example, reliability and authoritativeness of contents of wikis (Korfiatis et al. 2006). Other example include, machine learning techniques to user modeling and embedding sentiments in social media (Liu et al. 2006), and illicit content identification (Yang et al. 2012). For more examples see Table 6.

The online version of the original article can be found under doi:10.1007/s11192-012-0831-5.

G. F. Khan $(\bowtie)$

School of Industrial Management, Korea University of Technology and Education (KoreaTECH), 1600 Chungjol-ro Byungcheon-myun, Cheonan City 330-708, South Korea

e-mail: gohar.feroz@gmail.com 
Table 5 Bursting topic words for SMS research

\begin{tabular}{|c|c|c|c|c|c|c|c|}
\hline Burst & Weight & Start & End & Burst & Weight & Start & End \\
\hline \multicolumn{8}{|c|}{ Organization aspect of SMS } \\
\hline Organ & 4.50 & 2007 & 2007 & Collabor & 4.10 & 2005 & 2006 \\
\hline Competit & 2.25 & 2008 & 2008 & Practition & 2.25 & 2011 & \\
\hline Coordin & 1.67 & 2010 & & Healthcar & 2.25 & 2011 & \\
\hline Busi & 1.15 & 2004 & 2004 & Polici & 2.35 & 2009 & 2009 \\
\hline Deliveri & 2.66 & 2009 & 2009 & Partner & 2.25 & 2008 & 2008 \\
\hline Project & 2.67 & 2006 & 2007 & Member & 3.32 & 2005 & 2005 \\
\hline Incent & 2.30 & 2008 & 2008 & Intern & 3.83 & 2004 & 2006 \\
\hline \multicolumn{8}{|c|}{ Non-organization aspect of SMS } \\
\hline Public & 2.97 & 2005 & 2007 & Mobil & 3.42 & 2009 & 2009 \\
\hline Believ & 2.50 & 2005 & 2006 & Vulner & 2.28 & 2006 & 2007 \\
\hline Actor & 3.09 & 2005 & 2007 & Involv & 2.36 & 2005 & 2005 \\
\hline Subgroup & 2.74 & 2004 & 2005 & Reciproc & 4.05 & 2010 & \\
\hline Relat & 2.97 & 2004 & 2004 & Cultur & 2.79 & 2006 & 2007 \\
\hline \multicolumn{8}{|c|}{ Technical aspect of SMS } \\
\hline Algorithm & 3.9 & 2008 & 2008 & Indic & 2.70 & 2005 & 2005 \\
\hline Exploratori & 2.35 & 2009 & 2009 & Extract & 4.91 & 2004 & 2005 \\
\hline Visual & 4.56 & 2004 & 2007 & Charact & 2.30 & 2009 & 2009 \\
\hline Heurist & 3.12 & 2008 & 2008 & Multidimen & 2.28 & 2005 & 2007 \\
\hline Discoveri & 2.63 & 2008 & 2008 & Dataset & 2.29 & 2011 & \\
\hline Natur & 2.61 & 2004 & 2006 & Equat & 2.30 & 2009 & 2009 \\
\hline Emot & 2.49 & 2010 & & Scienc & 4.76 & 2004 & 2006 \\
\hline Random & 2.77 & 2010 & & Properti & 2.93 & 2007 & 2007 \\
\hline Softwar & 3.68 & 2004 & 2007 & Differenti & 2.25 & 2011 & \\
\hline Infer & 2.58 & 2006 & 2007 & Threshold & 2.25 & 2008 & 2008 \\
\hline \multicolumn{8}{|c|}{ Social as a tool } \\
\hline Tool & 2.30 & 2004 & 2008 & Confer & 4.93 & 2005 & 2007 \\
\hline Authorship & 2.28 & 2005 & 2007 & Author & 2.97 & 2005 & 2007 \\
\hline Pattern & 2.27 & 2004 & 2005 & Central & 4.69 & 2005 & 2005 \\
\hline Correl & 2.40 & 2009 & 2009 & Hidden & 2.33 & 2007 & 2008 \\
\hline Record & 2.21 & 2006 & 2007 & Primarili & 2.30 & 2009 & 2009 \\
\hline Academ & 2.62 & 2007 & 2007 & Reveal & 2.23 & 2005 & 2006 \\
\hline Measur & 2.40 & 2007 & 2007 & Citat & 2.84 & 2004 & 2007 \\
\hline Scientist & 2.67 & 2004 & 2007 & Phenomena & 2.37 & 2010 & \\
\hline Expos & 2.69 & 2011 & & & & & \\
\hline
\end{tabular}

Weight-is the weight of this burst between its length. A higher weight could be resulted by the longer Length 